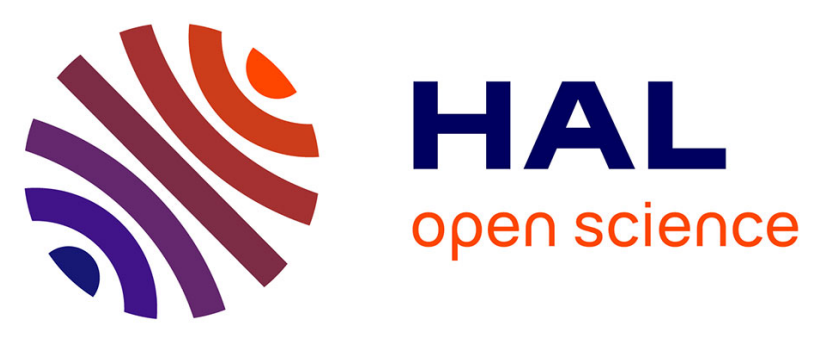

\title{
Assessing the Acceptability of Human-Robot Co-Presence on Assembly Lines: A Comparison Between Actual Situations and their Virtual Reality Counterparts
}

Vincent Weistroffer, Alexis Paljic, Philippe Fuchs, Olivier Hugues, Jean-Paul

Chodacki, Pascal Ligot, Alexandre Morais

\section{To cite this version:}

Vincent Weistroffer, Alexis Paljic, Philippe Fuchs, Olivier Hugues, Jean-Paul Chodacki, et al.. Assessing the Acceptability of Human-Robot Co-Presence on Assembly Lines: A Comparison Between Actual Situations and their Virtual Reality Counterparts. 23rd IEEE International Symposium on Robot and Human Interactive Communication (ROMAN), Aug 2014, Edinburgh, United Kingdom. 10.1109/ROMAN.2014.6926282 . hal-01079580

HAL Id: hal-01079580

https://hal-mines-paristech.archives-ouvertes.fr/hal-01079580

Submitted on 3 Nov 2014

HAL is a multi-disciplinary open access archive for the deposit and dissemination of scientific research documents, whether they are published or not. The documents may come from teaching and research institutions in France or abroad, or from public or private research centers.
L'archive ouverte pluridisciplinaire HAL, est destinée au dépôt et à la diffusion de documents scientifiques de niveau recherche, publiés ou non, émanant des établissements d'enseignement et de recherche français ou étrangers, des laboratoires publics ou privés. 


\title{
Assessing the Acceptability of Human-Robot Co-Presence on Assembly Lines: A Comparison Between Actual Situations and their Virtual Reality Counterparts
}

\author{
Vincent Weistroffer*, Alexis Paljic*, Philippe Fuchs*, Olivier Hugues*, \\ Jean-Paul Chodacki*, Pascal Ligot ${ }^{\dagger}$ and Alexandre Morais ${ }^{\dagger}$ \\ ${ }^{*}$ Mines ParisTech, Robotics Center - CAOR \\ ${ }^{\dagger}$ PSA Peugeot-Citroën
}

\begin{abstract}
(CIEEE 2014. This is the author's version of the work. It is posted here for your personal use. Not for redistribution. The definitive Version of Record was published in ROMAN 2014, http://dx.doi.org/10.1109/ROMAN. 2014.6926282.

Abstract-This paper focuses on the acceptability of humanrobot collaboration in industrial environments. A use case was designed in which an operator and a robot had to work sideby-side on automotive assembly lines, with different levels of co-presence. This use case was implemented both in a physical and in a virtual situation using virtual reality. A user study was conducted with operators from the automotive industry. The operators were asked to assess the acceptability to work side-by-side with the robot through questionnaires, and physiological measures (heart rate and skin conductance) were taken during the user study. The results showed that working close to the robot imposed more constraints on the operators and required them to adapt to the robot. Moreover, an increase in skin conductance level was observed after working close to the robot. Although no significant difference was found in the questionnaires results between the physical and virtual situations, the increase in physiological measures was significant only in the physical situation. This suggests that virtual reality may be a good tool to assess the acceptability of human-robot collaboration and draw preliminary results through questionnaires, but that physical experiments are still necessary to a complete study, especially when dealing with physiological measures.
\end{abstract}

\section{INTRODUCTION}

Robots are becoming more and more present in our everyday lives. Robots are now able to guide people inside museums, some can communicate and play with children, or even be used as therapeutic companions in hospitals. Those new applications for robots show that situations where humans and robots interact with each other are becoming more and more important. An even stronger level of interaction is reached when humans and robots have to collaborate on a specific task, especially in industrial environments such as assembly lines: humans and robots may have different roles and complementary skills. In this context, new kinds of robots have appeared that are able to work side-by-side with people without harming them. Although the functionalities of those new collaborative configurations have been well studied in the literature, no concrete studies have appeared to evaluate how the collaboration with robots is perceived and accepted by workers, at least in industrial environments. That is why this paper will not present any considerations about robot functionalities (robot control, movement planning), but will rather focus on operators' behavior towards human-robot configurations: a specific use case was designed to assess the acceptability of human-robot co-presence on assembly lines.

The second issue addressed in this paper is the relevance of the use of virtual reality to study the acceptability of human-robot collaboration. For practical and economic reasons, some studies on the acceptability of human-robot interaction have used images or videos of robots as stimuli to the users. Their impressions could then be analyzed to design an acceptable physical robot. Other studies have used virtual reality scenarios to incorporate more interaction with the robots in the simulation. However, the impressions of people towards images, videos or simulations of robots may be different from their experience in real situations with physical robots. This paper tries to shed some light on those considerations by comparing people's reactions when collaborating with an industrial robot both in a physical situation and its virtual reality counterpart.

\section{RELATED WORK}

\section{A. Human-Robot Collaboration}

Human-robot interaction is a wide field in which researchers study how people interact with robots, especially in a social manner [1]. Those robots can be used as domestic assistants at home [2], guides in museums [3], companions for children [4] or for the elderly [5] in hospitals. Humanrobot collaboration implies more interaction between people and robots: they have to work together on a common task in order to reach a common goal [6]. Such a collaboration betwen a human and a robot has already been studied with social robots, for example with Leonardo [7] for a buttonpressing task or the mobile robot helper [8] to handle an object with a human. More complex scenarios, more related to the industry, have arisen, such as solving a construction task collaboratively with a human (JAST robot [9]) or helping a human co-worker to collect parts and put them into bins [10].

In industrial environments, human-robot collaboration has taken increasing importance: industrial robots can provide complementary skills to human co-workers and assist them in difficult, tiring and hazardous tasks. Such robots have to work alongside humans in a safe way without harming them. New 
industrial robots have been designed to comply with those needs, such as the Universal Robot UR5 ${ }^{1}$, the Kuka LWR [11], the Kuka $\mathrm{MRK}^{2}$ or the recent Baxter robot [12] from Rethink Robotics. The applications of those robot assistants in the industry are diverse: fetching and carrying objects, like the rob@work [13], or assisting humans with handling and assembling parts, like the PowerMate [14].

\section{B. Acceptability of Human-Robot Collaboration}

Although those new kinds of robots already exist in industrial environments and a large part of the literature has been spent on their functionalities, especially by making them physically safe for human-robot interaction and collaboration [15], little has been done to study how those robots are perceived and accepted in the work environment of people.

In the field of social robotics, the question of the acceptability to interact with robots has been well studied. De Graaf and Ben Allouch [16] recently provided an interesting exploration of the influencing variables for the acceptance of social robots. One of the most studied factor in humanrobot interaction is probably robot appearance and especially anthropomorphism. Mori's Uncanny Valley [17] asserts that a robot's anthropomorphism tends to increase its familiarity among people, until a certain point is reached where strange details, almost human-looking, may actually repel people. A large number of studies on robot appearance were conducted to confirm or discredit this effect [18] [19]. Another factor influencing the acceptability to interact with robots is the nature of their movements: several studies tried to discern what were the best robot movement profiles to interact with people [20] [21] [22]. The acceptability of human-robot collaboration may also be influenced by user characteristics, such as age, gender or culture.

In the field of industrial robotics, factors such as robot appearance and movements may still have an influence on the acceptability of human-robot collaboration. Hinds et al. [10] focused on professional service robots and the influence of a robotic coworker's appearance and role on a simple partscollecting task. Zanchettin et al. [21] studied the influence of a robotic manipulator's movements while sharing a working environment with a human. Weistroffer et al. [22] compared different industrial robot arms and movement profiles while exchanging parts with a human. Karwowski and Rahimi [23] studied the influence of robot size and robot speed on the operators' willingness to enter a robot's working area.

However, additional factors probably have to be taken into account when collaborating with industrial robots, such as time distribution and space distribution between the human and the robot [14]. Indeed, robot appearance and movements are likely to assume less importance as people are generally focused on their own task, even when working alongside robots. Time distribution and space distribution are actually little studied as factors in the acceptability of human-robot collaboration. In this paper, a specific industrial use case

\footnotetext{
${ }^{1}$ http: //www. universal-robots.com

${ }^{2}$ http://www.mrk-systeme.de
}

was designed to evaluate the influence of human-robot copresence on the acceptability to work with robots.

\section{Virtual Reality}

The study of human-robot interaction uses either actual scenarios, in which people interact directly with robots, or pre-recorded scenarios, in which people are shown different images or videos of robots. When dealing with human-robot collaboration, a high level of interaction is required in the scenarios: images and videos are not sufficient to account for this need. That is why virtual reality has been used in some cases to place people in virtual environments and to make them interact with virtual robots. For example, De Santis et al. [24] used virtual reality to study the usability of robotic arms mounted on a wheelchair. Nonaka et al. [25] used virtual situations to evaluate the influence of robot movements on people's perception. Weistroffer et al. [22] used virtual industrial robots to study the influence of their appearance and movements on people's acceptability to collaborate with them.

However, little is known about the relevance to use virtual reality situations as counterparts of real physical situations: people are likely to behave differently in front of virtual robots (probably feeling less threat). The question here is how strongly can we rely on virtual reality simulations results to be reproducible in equivalent physical situations. Inoue et al. [26] compared human-robot interaction tasks (handling objects and moving close to humans) using the same physical and virtual situations and showed that human psychological states were the same with the virtual and physical robots. However, the users were mainly passive in front of the robots (there was no physical interaction and collaboration with the robots) and their physiological states were only measured through subjective questionnaires. Those limitations probably call for deeper analysis about the relevance to use virtual reality, especially when physical contact is possible between humans and robots and when physiological measures are used to assess people's affective state.

\section{Study Objectives}

This paper focuses on two specific issues: evaluating the acceptability of human-robot co-presence in industrial environments, and assessing the relevance to use virtual reality experiments in this evaluation. In this context, a specific use case was set up, largely inspired by industrial assembly lines, in which an operator and a robot had to work side-by-side on a car door. This use case was implemented both in a physical environment (with an actual robot and actual assembly tasks) and in a virtual environment. A user study was conducted in order to compare users' reactions.

\section{TASK DESCRIPTION}

\section{A. Current Assembly Task}

In the context of human-robot collaboration in an industrial environment, the situation we decided to study is a car door assembly. One of the necessary operations during this 
assembly is the setting of the sealing sheet. The current task in car plants requires the operators to put a sealing sheet on the door, to ensure it is well positioned and to permanently stick it to the door. This last operation requires the operators to take a metallic pattern (the shape of the sheet) above their heads, to put it on the sheet and to roll the glue lines of the sheet with a caster at the edges of the pattern (see Fig. 1). This task can be tiring for the operators since they have to look for the pattern above their heads (uneasy position) and they have to manipulate the caster (uneasy movements of the wrist). Work quality is also at stake here since this operation is not correctly reproducible. That is why a new assembly task was considered, with a robot performing the caster-rolling task on the sheet while the operator is free to perform operations next to the robot.

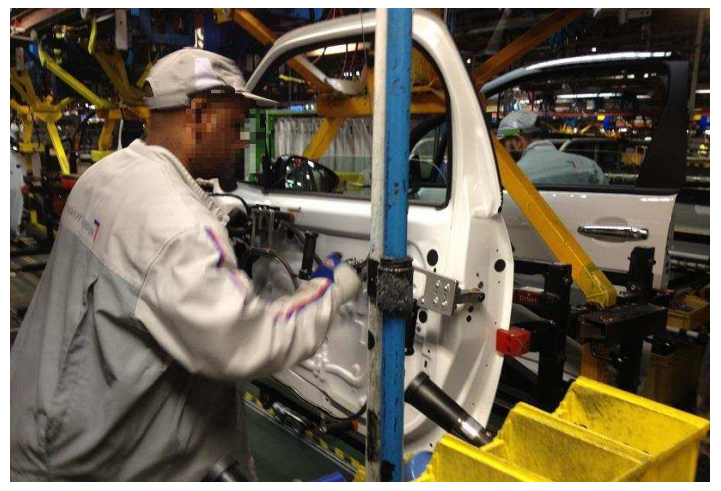

Fig. 1. Current task on assembly lines

\section{B. New Assembly Task}

In this new assembly task, the operator and the robot are placed next to each other: the operator first places the sheet on the door and the robot then has to roll the caster around the edges of the sheet to fix it permanently (see Fig. 2). This configuration occurs while the doors are in movement on assembly lines. To optimize assembly time and be as close as possible to a real situation, the operator also has to perform additional operations on the door while the robot is working: assembling adhesive shutters, plastic clips, the window scraper, a body of cables and the door joint. The order of those operations was fixed considering the movement direction of the doors on the assembly lines (from left to right when facing the doors) and the configuration with the robot. It is shown in Fig. 3.

Such a use case has already been shown by BMW [27] with a Universal UR10 robot, but little information is available about it: it seems the robot performed its task alone without any interaction with humans. In our situation, we wanted the operator and the robot to be co-workers and to work side-by-side, enabling them to interact and to touch each other, for example when the operator was late in his work. This situation was representative of human-robot copresence (rather than collaboration): the operator and the robot had to share the same physical space.

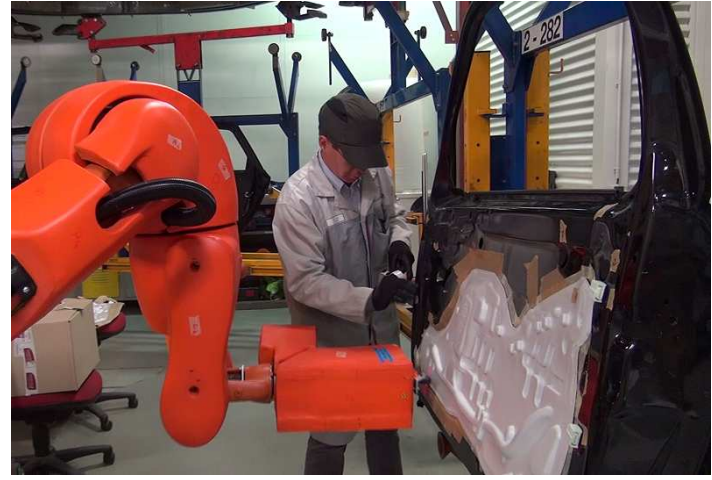

Fig. 2. New assembly task with human-robot co-presence
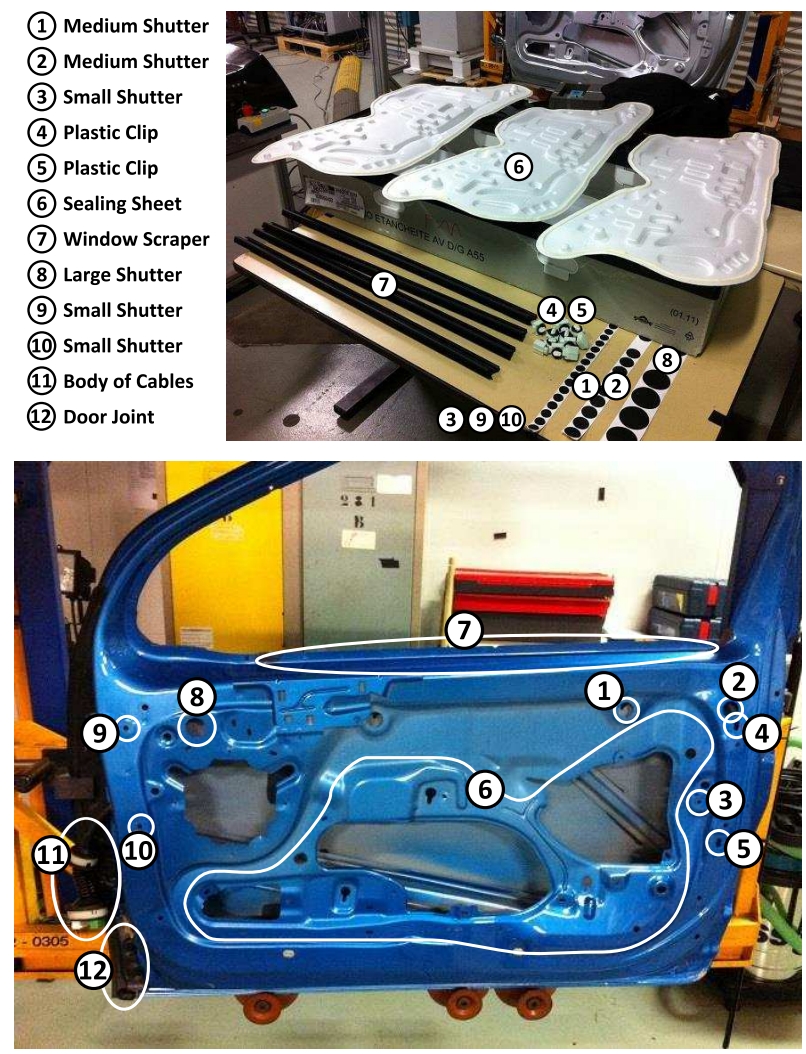

Fig. 3. Operations order, assembly parts and their locations on the door

This scenario was implemented both in a physical and in a virtual environment (see Fig. 4). The physical environment reproduced the industrial context by having a prototype of assembly line. For space reasons, the assembly line was shaped in a loop. The longest straight path along the assembly line was approximately 6 meters. Four car doors (front right door of a Citroën DS3 car model) were positioned next to each other on the line. The speed of the line was approximately $0.07 \mathrm{~m} / \mathrm{s}$ (representing 40 cars/hour) and could be modified if necessary. The robot was positioned in the middle of the loop.

\section{Human-Robot Configuration}

Since one of our aims was to study the acceptability of human-robot co-presence on assembly lines, one necessary 

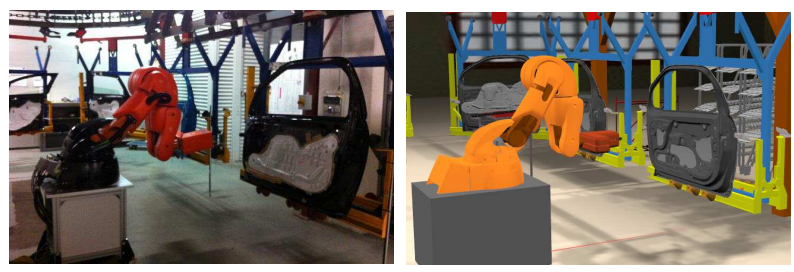

Fig. 4. The physical (left) and virtual (right) environments

factor was the distance between the operator and the robot. In that context, two conditions of human-robot co-presence were chosen: one where the operator works far away from the robot $\left(\mathrm{P}_{\mathrm{f}}\right)$ and the other where the operator works close to the robot $\left(\mathrm{P}_{\mathrm{C}}\right)$. The size of the working areas was the same for both conditions $(1.8 \mathrm{~m})$ but they were located at different distances from the robot: for $\mathrm{P}_{\mathrm{f}}$, it began at $3.9 \mathrm{~m}$ from the robot, and for $\mathrm{P}_{\mathrm{C}}$ it began at $3 \mathrm{~m}$ (see Fig. 5). The operator could begin to work on the door once it was fully inside the working area and had to stop when the door completely left the area (even if all the tasks were not completed). The operators were given start and stop notifications by the coordinators.

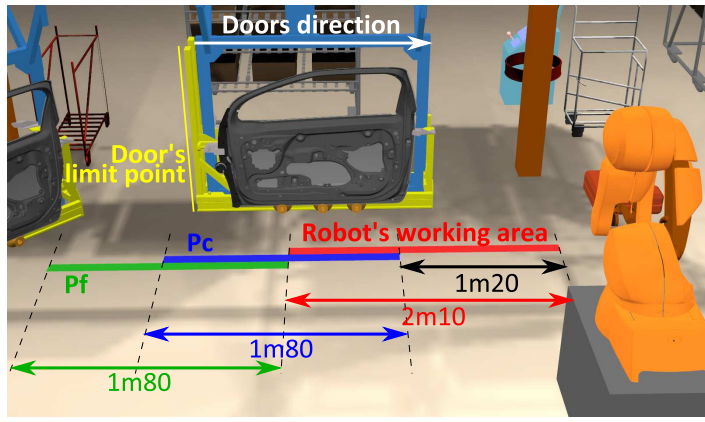

Fig. 5. The two configurations of human-robot co-presence: far and close

\section{Robot's Behavior}

The robot that was chosen for the task was a Kuka KR5SI: this is a Kuka KR5 ARC HW allowing safe interaction with humans thanks to the integration of additional sensors by the company MRK-Systeme ${ }^{3}$. Indeed, the robot was supplemented with damping safety pads around its initial structure, in which were located proximity capacitive sensors and tactile sensors to detect any collision. The robot could detect the proximity of an operator at a distance of around $10 \mathrm{~cm}$ from its outer surface. Whenever such a detection happened, a sound signal was triggered and the robot stopped its current activity (the assembly line went on); the sound signal vanished and the robot resumed its task once the human presence went farther from the robot. If a strong hit occured on the safety pads, an emergency stop was triggered: the robot stopped as well as the assembly line.

For the robot to be aware of the assembly line progress, a laser sensor was placed above and behind the robot to detect

\footnotetext{
${ }^{3}$ http: //www.mrk-systeme.de
}

the arrival of a specific door. Moreover, to detect the position of the edges of the sealing sheet on the door, another laser sensor was placed inside the robot head. The robot head was also equipped with a caster to perform its operation on the door. Since the topic of this paper is not about robot functionalities, this will not be described any further.

\section{E. Virtual Environment}

The virtual environment was a faithful reproduction of the physical one (see Fig. 4). Exact 3D models of the actual assembly line, including the doors, the robot and the assembly parts, were used at real scale in the virtual environment. The virtual assembly parts could be taken by approaching one's virtual hands from them and could be assembled on the door by moving them to their correct locations (highlighted in red). The operators could use both hands to manipulate assembly parts: one hand was sufficient for small parts, while two hands were required for the sealing sheet and the window scraper.

The virtual scenario was implemented within the Virtools VR software ${ }^{4}$. The virtual environment was projected inside a 3-side CAVE using active stereoscopy: the front and the floor screens were 4 meters wide, and an additional screen on the right enabled the operators to observe the robot next to them (see Fig. 6). The movements of the users were tracked thanks to ARTrack cameras: 3D trackers were positioned on their head, their hands and each side of their back. The hand trackers enabled the operators to manipulate the virtual assembly parts, while all the trackers were used for proximity detection with the virtual robot. If such a detection occured $(10 \mathrm{~cm})$, the same sound signal than in the physical environment was triggered.

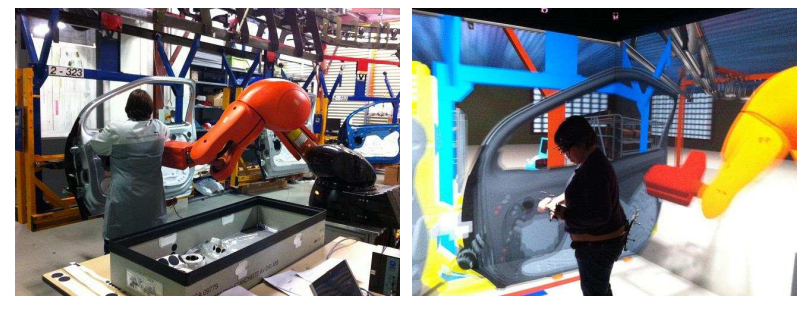

Fig. 6. A user in the physical environment and inside the CAVE

\section{USER STUDY}

\section{A. Aims of the Study}

The aims of this study were twofold: evaluating the acceptability of human-robot co-presence on assembly lines, and assessing the relevance to use virtual reality in this evaluation. In this context, a within-subject study was performed with operators from car assembly lines. Two independent variables were taken into account: the situation $\mathrm{S}$ of the test (physical $S_{p}$ or virtual $S_{V}$ ) and the co-presence $P$ with the robot (far $\mathrm{P}_{\mathrm{f}}$ or close $\mathrm{P}_{\mathrm{c}}$ ).

\footnotetext{
${ }^{4}$ http: //www. 3ds.com/products-services/3dvia/ 3dvia-virtools/
} 


\section{B. Protocol}

For each subject, the test lasted a whole day: one half-day for the physical situation and the other half-day for the virtual situation. Half of the users began with the physical situation (morning) and ended with the virtual situation (afternoon), while the other half did the opposite. For each half-day, the order of conditions $\mathrm{P}_{\mathrm{f}}$ and $\mathrm{P}_{\mathrm{C}}$ was randomised.

At the beginning of each half-day, the operators were given a document with information about the operations to perform on the doors (either physical or virtual). They were given as much time as they wanted to learn the operations and train on a single door (a physical one for $S_{p}$ and a virtual one for $\mathrm{S}_{\mathrm{V}}$ ): for this static training step, the doors were not moving and the robot was off. Once the operators were ready, some time was given to show how the robot was performing its task and was behaving next to humans. After this description, the test began: for each condition, the operators had to complete four cycles of doors (one cycle being a four-door procession). The two first cycles were mainly used for training purposes while the two last ones were used for actual data acquisition.

Questionnaires were given and physiological measures were taken at different moments of the test: they are described in Section IV-C. An overview of the whole protocol is shown in Table I, while Table II shows the protocol for a single condition.

TABLE I

The Whole Protocol of the User Study

\begin{tabular}{|l|c||c|c||c|}
\cline { 2 - 5 } \multicolumn{1}{c|}{} & \multicolumn{4}{c|}{ Morning $\mathrm{S}_{\mathrm{V}}$} \\
\hline Step & Training & Step 1 & Step 2 & Quest. \\
Condition & $\varnothing$ & $\mathrm{P}_{\mathrm{C}}$ & $\mathrm{P}_{\mathrm{f}}$ & $\mathrm{Q}_{\mathrm{S}}$ \\
\cline { 2 - 5 } \multicolumn{4}{c|}{ Afternoon $\mathrm{S}_{\mathrm{p}}$} \\
\hline Step & Training & Step 1 & Step 2 & Quest. \\
Condition & $\varnothing$ & $\mathrm{P}_{\mathrm{f}}$ & $\mathrm{P}_{\mathrm{C}}$ & $\mathrm{Q}_{\mathrm{S}}$ \\
\hline
\end{tabular}

TABLE II

The Protocol for a Single Condition

\begin{tabular}{|l|c||c|c|c|c||c|}
\cline { 2 - 7 } \multicolumn{1}{c|}{ Cycle } & $\varnothing$ & Cycle 1 & Cycle 2 & Cycle 3 & Cycle 4 & Quest. \\
Doors & $\varnothing$ & $1-4$ & $1-4$ & $1-4$ & $1-4$ & $\mathrm{Q}_{\mathrm{p}}$ \\
Physio. & $\phi_{0}$ & $\phi_{1}$ & $\phi_{2}$ & $\phi_{3}$ & $\phi_{4}$ & ${ }^{2}$ \\
\hline
\end{tabular}

\section{Measures}

To compare each condition and assess the acceptability of human-robot collaboration from the operators' point of view, several data were collected. As was proposed by Weistroffer et al. [22], a set of questionnaires was used to gather the users' subjective impressions, and physiological measures were taken to assess their affective state. Performance data were also collected during the tasks.

1) Questionnaires: Bartneck et al. [28] provided an interesting survey of questionnaires related to human-robot interaction and proposed their own standard ones, mainly focused on social robots. We took inspiration from them and wrote our own questionnaires to be more representative of the industrial context and of the considerations of the study: we added questions related to safety, utility, efficiency and acceptability, and questions about human-robot co-presence, related to the distance to the robot.

The users were given a questionnaire $Q_{p}$ after each condition (see Table II): it consisted in 6-point Likert scales to assess their subjective impressions about the activity and co-presence with the robot. They were also given a more global questionnaire $\mathrm{Q}_{\mathrm{S}}$ at the end of each half-day (see Table I), to assess their level of comfort with the operations and the use of the system. The whole day eventually ended with open questions: the operators could give any remarks about the robot, the co-presence configuration or the test itself.

2) Physiological Measures: Kulić and Croft [20] were the first ones to use physiological measures to assess the affective states of users interacting with robots. For their experiments, they used heart rate, skin conductance and facial muscle contraction as indicators of the users' affective state while observing robot movements.

For our study, we measured heart rate and skin conductance thanks to a physiological monitoring system from Biopac Systems ${ }^{5}$ with a Bionomadix PPG (Photoplethysmogram) and EDA (Electrodermal Activity) amplifier. PPG signal provided information on the subject's pulse rate (PR), while EDA signal measured the skin conductance level (SCL). It was not possible to record the operators physiological measures while working: the measures were taken on the fingertips and the signals were disturbed by hand movement and object prehension, making the analysis impossible. Therefore, the measures were taken at specific moments of the test while the operators were sitting for about 3 minutes (see Table II): at the beginning of each condition $\left(\phi_{0}\right)$ and right after completing each cycle $\left(\phi_{1}, \phi_{2}, \phi_{3}, \phi_{4}\right)$.

For the off-line analysis, for each measure, the mean PR and the mean SCL were computed over one minute (during the rest period for $\phi_{0}$, and following rightaway the activity for $\left.\phi_{1}, \phi_{2}, \phi_{3}, \phi_{4}\right)$. This resulted in 20 indicators per user $(5$ measures $\times 4$ conditions) both for PR and SCL. For each user, the minimum $\mathrm{m}$ and maximum $\mathrm{M}$ values were found among all the indicators and each indicator was then normalized between $\mathrm{m}$ and $\mathrm{M}$ (giving a value between 0 and 1). This normalization enabled the comparison of physiological results between users.

3) Performance Data: To complement the results obtained through questionnaires and physiological measures, we also gathered performance data from the activity on the doors. We were interested in the number of non-completed operations, the number of proximity detections by the robot (sound signal) and the task duration (from the first part assembled on the door, to the last one or to the exit of the door from the operator's area). Those data were collected for each door. In the physical situation, they were gathered off-line thanks to video recordings of the tests. In the virtual situation, those data were automatically saved through the software (additional data were also gathered).

\footnotetext{
${ }^{5}$ http: //www.biopac.com
} 


\section{Population}

For this user study, the population was chosen from a set of operators working on assembly lines in the car industry. Eight operators participated in this user study (the two first ones were mainly used to adjust the test settings and were not taken into account in the results): one woman and seven men, with an average age of 40 . It was really important to have participants who were from the industrial field to get relevant results. It was however not possible to get as large a number of participants as we wanted, mainly due to the availabality of operators and time constraints (the test lasted a whole day). Experiments are still conducted in order to increase the population size.

\section{RESULTS}

\section{A. Performance Data}

The performance data consisted in the number of noncompleted operations, the number of proximity detections with the robot and the task duration. They were all computed for each door. Fig. 7 shows the average over all the doors and all the users.

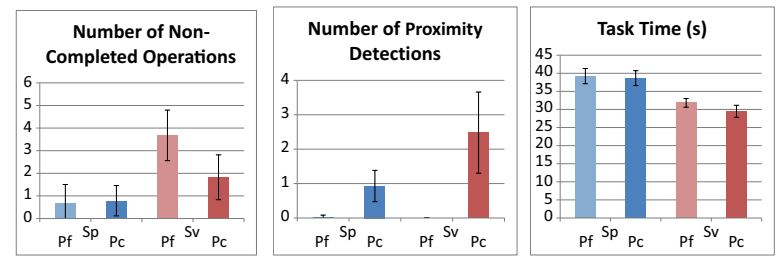

Fig. 7. Users' performance data

On the whole, the operators did not have difficulties in completing their operations, both in the physical and virtual situations. They were however less used to virtual operations than physical ones: that is why the number of non-completed operations was higher in $S_{V}$ (more than two) than in $S_{p}$ (less than one). One possible explanation is the lack of haptic feedback for $S_{V}$. Moreover, the average task time was lower in $S_{V}$ than in $S_{p}$ : even if the operators were not used to it, the virtual operations took less time to perform than physical ones.

When working in the virtual situation, the operators behaved differently and were less aware of the virtual robot presence next to them: either because they could not sense it haptically, or because they could not see it in their peripheral vision (because of the frame of the stereoscopic glasses), or even because they could not properly hear the robot movements next to them (only the proximity sound signal was simulated). The operators often became conscious of the virtual robot proximity only once they heard the sound signal, thus explaining the higher number of proximity detections in $\mathrm{S}_{\mathrm{V}} \mathrm{P}_{\mathrm{c}}$ compared to $\mathrm{S}_{\mathrm{p}} \mathrm{P}_{\mathrm{C}}$.

Finally, the reduced awareness of the robot presence in $\mathrm{S}_{\mathrm{V}}$ probably made the operators more relaxed, and this relaxation was all the more important since the operators were far from the robot $\left(\mathrm{S}_{\mathrm{V}} \mathrm{P}_{\mathrm{f}}\right)$. Being more relaxed, the operators spent more time on their operations and became late: indeed, the number of non-completed operations and the task duration were significantly higher for $\mathrm{S}_{\mathrm{V}} \mathrm{P}_{\mathrm{f}}$ than for $\mathrm{S}_{\mathrm{V}} \mathrm{P}_{\mathrm{C}}$. A Wilcoxon test was performed for the number of noncompleted operations $(\mathrm{W}=854, \mathrm{p}<0.001)$, while an ANOVA was performed for the task duration $(\mathrm{F}=18, \mathrm{p}<0.001)$.

\section{B. Questionnaires}

1) Co-Presence Questionnaire $Q_{p}$ : The results of the copresence questionnaire $\mathrm{Q}_{\mathrm{p}}$ are shown in Fig. 8 (for each question, the average answer over all the users is taken). Wilcoxon tests were performed to assess significant differences in the results.

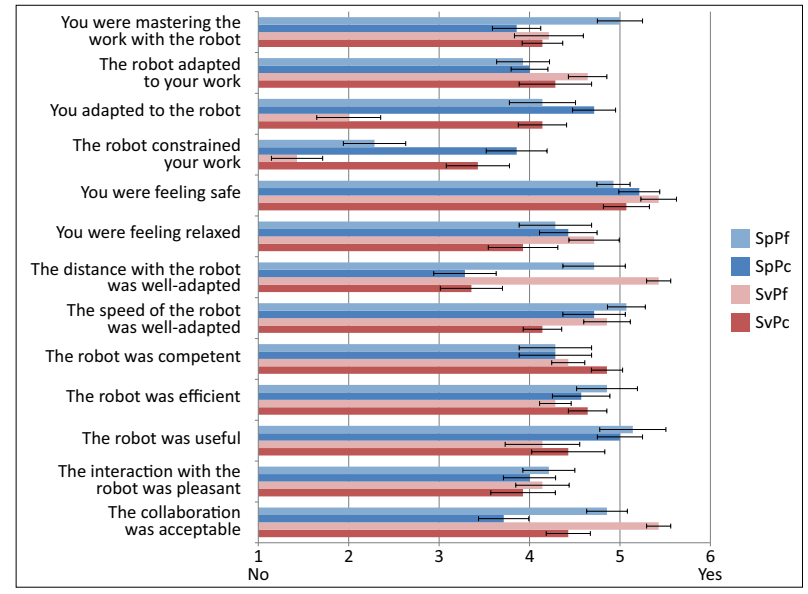

Fig. 8. Results of the $\mathrm{Q}_{\mathrm{p}}$ questionnaire

On the whole, the co-presence with the robot was found more acceptable (last question) in the far condition than in the close condition, both in the physical and virtual environments, even if no significant difference was shown. Three main reasons appear to explain this trend.

First, the distance to the robot was found more adapted in the far condition in both situations: Wilcoxon tests showed a significant difference between $\mathrm{S}_{\mathrm{p}} \mathrm{P}_{\mathrm{f}}$ and $\mathrm{S}_{\mathrm{p}} \mathrm{P}_{\mathrm{c}}(\mathrm{W}=51.5$, $\mathrm{p}=0.04)$, and between $\mathrm{S}_{\mathrm{V}} \mathrm{P}_{\mathrm{f}}$ and $\mathrm{S}_{\mathrm{V}} \mathrm{P}_{\mathrm{c}}(\mathrm{W}=56.5, \mathrm{p}=0.007)$.

Secondly, the operators found that the robot constrained their work more in the close condition than in the far condition: Wilcoxon tests showed a significant difference between $\mathrm{S}_{\mathrm{p}} \mathrm{P}_{\mathrm{f}}$ and $\mathrm{S}_{\mathrm{p}} \mathrm{P}_{\mathrm{c}}(\mathrm{W}=12.5, \mathrm{p}=0.04)$, and between $\mathrm{S}_{\mathrm{V}} \mathrm{P}_{\mathrm{f}}$ and $\mathrm{S}_{\mathrm{V}} \mathrm{P}_{\mathrm{c}}(\mathrm{W}=8, \mathrm{p}=0.006)$.

Finally, the operators had to adapt to the robot more in the close condition than in the far one: Wilcoxon tests showed a significant difference only between $\mathrm{S}_{\mathrm{V}} \mathrm{P}_{\mathrm{f}}$ and $\mathrm{S}_{\mathrm{V}} \mathrm{P}_{\mathrm{C}}(\mathrm{W}=7$, $\mathrm{p}=0.007$ ).

Some significant differences appeared between the physical and virtual situations: for the far condition, the robot induced less constraints and less necessity to adapt in the virtual situation than in the physical one $(\mathrm{W}=50, \mathrm{p}=0.004$; $\mathrm{W}=58, \mathrm{p}=0.005$; respectively). This is again explained by the fact that, in the virtual situation, the operators were less aware of the presence of the robot.

No significant difference was found with the other questions. 
2) Situation Questionnaire $Q_{S}$ : The results of the situation questionnaire $\mathrm{Q}_{S}$ are shown in Fig. 9. Wilcoxon tests were performed to assess significant differences in the results.

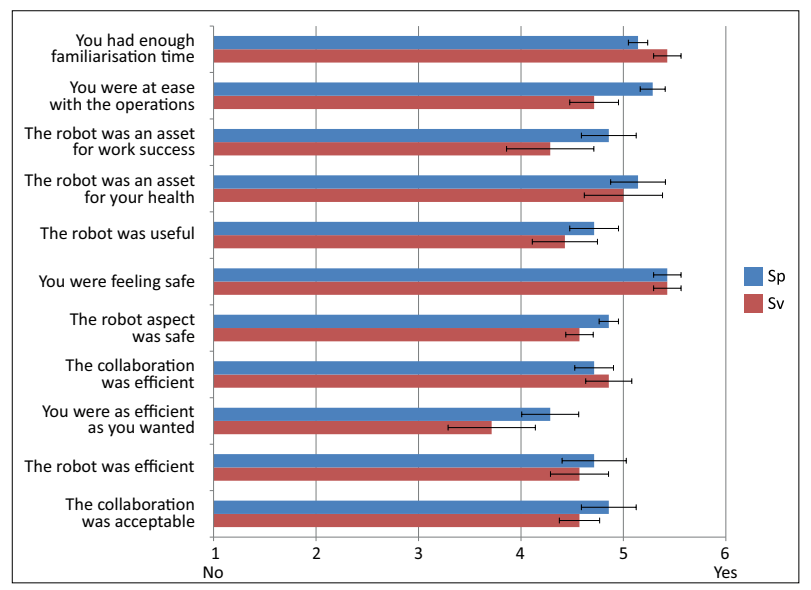

Fig. 9. Results of the $\mathrm{Q}_{\mathrm{S}}$ questionnaire

On the whole, no significant difference was found in the questionnaire between the physical and virtual situations. However, it can be noted that the operators were feeling more at ease and efficient with the operations in the physical situation: this is understandable since they were not especially used to virtual reality settings.

\section{Physiological Measures}

The physiological measures consisted in the users' average pulse rate (PR) and skin conductance level (SCL) right after the activity. For each condition, four measures were taken into account: the normalized $\phi_{1}, \phi_{2}, \phi_{3}, \phi_{4}$. The results are shown in Fig. 10 (for each condition, the average over $\phi_{1}, \phi_{2}, \phi_{3}, \phi_{4}$ and over all the users was taken). Two-way ANOVAs were performed to assess significant differences in the results, situation (physical or virtual) and distance to the robot (far or close) being the independent variables.

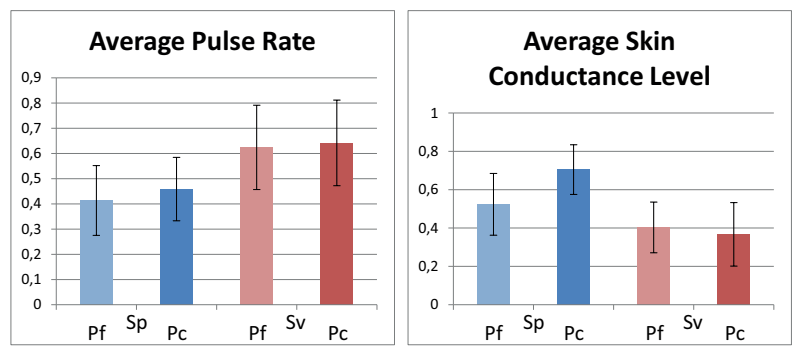

Fig. 10. Results of the physiological measures

Both for pulse rate and skin conductance level, a significant influence of the situation was found $(\mathrm{F}=3.28, \mathrm{p}=0.08$ for PR; F=5.54, $\mathrm{p}=0.03$ for SCL). Those differences between situations are certainly inherent to the fact that the two situations occured at different moments of the day (two halfdays). The influence of the distance to the robot (between $\mathrm{P}_{\mathrm{f}}$ and $\left.\mathrm{P}_{\mathrm{C}}\right)$ is more important in the analysis.
No significant influence of the distance to the robot was found for the average pulse rate. However, the average pulse rate seems higher after working close to the robot compared to far from it, especially in the physical situation. This correlates with the questionnaires results: by working closer to the robot, the operators were more constrained in their work, had to adapt to the robot and had to speed their activity, thus inducing an increase in pulse rate. This trend was less relevant in the virtual situation.

A significant influence of the interaction between situation and distance to the robot was found for the average skin conductance level $(\mathrm{F}=8, \mathrm{p}=0.009)$. Indeed, in the physical situation, the average SCL was significantly higher after working close to the robot than far from it $(\mathrm{F}=4.6, \mathrm{p}=0.04)$, while there was no significant difference in the virtual situation. In the physical situation, the operators were more stressed when working close to the robot, although they did not mention it in the questionnaires. This stress occured because the robot was a constraint and the operator had to adapt to it in order to finish in time. However, this trend did not appear in the virtual situation: the operators were less aware of the robot presence, they probably felt less threat from the virtual robot and considered their activities less seriously.

On the whole, working close to the robot seems to have made the operators speed their activities, thus inducing an increase in their pulse rate and in their level of stress. This trend however did not appear in the virtual situation.

\section{CONCLUSION}

This paper focused on the acceptability of human-robot collaboration in industrial environments. A use case was implemented in which an operator and a robot had to work in co-presence on automotive assembly lines. A user study was conducted to assess the acceptability of human-robot copresence at different distances to the robot. This user study included a physical situation and its virtual counterpart to evaluate the relevance to use virtual reality in the study of acceptability.

Both in the physical and virtual situations, the co-presence seemed more acceptable when working far from the robot. Indeed, the questionnaires results showed that the distance was less adapted in the close condition because the robot was perceived as a constraint and the operators had to adapt their work to the robot. This correlates with the results on physiological measures, which showed an increase in skin conductance level after working close to the robot compared to far from it (only in the physical situation). However, working far from the robot may also decrease the operator's efficiency (as shown in the virtual situation), since the operators seemed to have more time to complete the operations. These results show that questionnaires and physiological measures are good indicators in the overall study of the acceptability of human-robot collaboration.

At first glance, virtual reality seems to be a relevant tool to study the acceptability of human-robot collaboration: the situation (physical or virtual) did not have any influence on 
the questionnaires results, except concerning the efficiency of the operators (less used to virtual reality systems). However, the tendency of physiological measures to increase after working close to the robot, visible in the physical situation, did not appear in the virtual one. This is probably due to a diminished feeling of presence in the virtual environment: the operators were less aware of the virtual robot presence, either haptically or visually, and were feeling more relaxed.

Although the non-reproducibility in the physiological results emphasizes the necessity to perform experiments in physical situations, these results show that virtual reality may be an interesting tool in the study of the acceptability of human-robot collaboration. The questionnaires results were globally the same in the physical and virtual situations: in future use cases, questionnaires results obtained through virtual situations could be used to draw interesting preliminary conclusions on acceptability before the design of actual physical situations. Virtual situations also have the possibility to easily test additional hypothesis that may not be feasible in physical ones. In our use case, we believe that having the operator and the robot work side-by-side, especially in the virtual situation, diverted the operators' awareness of the robot presence: the operators focused more on their task than on the robot. In the future, we intend to perform additional studies to overcome this situation, with use cases in which an operator and a robot face each other and have to cooperate with each other by exchanging assembly parts (human-robot co-action). We still plan to conduct user studies both in physical and virtual situations to further evaluate the relevance to use virtual reality in the study of the acceptability of human-robot collaboration.

\section{ACKNOWLEDGMENT}

The authors wish to thank the operators who gave their time to participate in the study. This research benefited from the support of the Chair 'PSA Peugeot Citroën - Robotics and Virtual Reality', led by MINES ParisTech and supported by PEUGEOT S.A. The partners of the Chair cannot be held accountable for the content of this paper, which engages the authors' responsibility only.

\section{REFERENCES}

[1] T. W. Fong, I. Nourbakhsh, and K. Dautenhahn, "A survey of socially interactive robots," Robotics and Autonomous Systems, vol. 1, pp. 143166, 2003.

[2] C. Parlitz, M. Hägele, P. Klein, J. Seifert, and K. Dautenhahn, "Careo-bot 3 - rationale for human-robot interaction design," in Proceedings of the 39th International Symposium on Robotics (ISR), 2008.

[3] S. Thrun, M. Bennewitz, W. Burgard, A. Cremers, F. Dellaert, D. Fox, D. Hähnel, C. Rosenberg, N. Roy, J. Schulte, and D. Schulz, "Minerva: a second-generation museum tour-guide robot," in Proceedings of the 1999 IEEE International Conference on Robotics and Automation, vol. 3, 1999, pp. 1999-2005.

[4] K. Dautenhahn and I. Werry, "A quantitative technique for analysing robot-human interactions," in IEEE/RSJ International Conference on Intelligent Robots and Systems, vol. 2, 2002, pp. 1132-1138.

[5] T. Shibata, K. Wada, Y. Ikeda, and S. Sabanovic, "Cross-cultural studies on subjective evaluation of a seal robot." Advanced Robotics, vol. 23, no. 4, pp. 443-458, 2009.

[6] A. Bauer, D. Wollherr, and M. Buss, "Human-robot collaboration: a survey," International Journal of Humanoid Robotics, vol. 5, no. 1, pp. 47-66, 2008.
[7] C. Breazeal, A. G. Brooks, J. Gray, G. Hoffman, C. D. Kidd, H. Lee, J. Lieberman, A. Lockerd, and D. Chilongo, "Tutelage and collaboration for humanoid robots." International Journal of Humanoid Robotics, vol. 1, no. 2, pp. 315-348, 2004.

[8] K. Kosuge, M. Sato, and N. Kazamura, "Mobile robot helper," in Proceedings of the 2000 IEEE International Conference on Robotics and Automation, vol. 1, 2000, pp. 583-588.

[9] M. E. Foster, M. Rickert, and M. Braun, "The JAST collaborative human-robot dialogue system," in Proceedings of CogSys II (Poster session), Nijmegen, NL, Apr. 2006.

[10] P. J. Hinds, T. L. Roberts, and H. Jones, "Whose job is it anyway? a study of human-robot interaction in a collaborative task," Human-Computer Interaction, vol. 19, no. 1, pp. 151-181, 2004.

[11] S. Haddadin, M. Suppa, S. Fuchs, T. Bodenmüller, A. Albu-Schäffer, and G. Hirzinger, "Towards the robotic co-worker," in International Symposium on Robotics Research, vol. 1, 2009, pp. 261-282.

[12] C. Fitzgerald, "Developing Baxter," in 2013 IEEE International Conference on Technologies for Practical Robot Applications (TePRA), 2013, pp. 1-6.

[13] E. Helms, R. Schraft, and M. Hägele, "rob@work: Robot assistant in industrial environments," in Proceedings of the 11th IEEE International Workshop on Robot and Human Interactive Communication, 2002, pp. 399-404.

[14] R. Schraft, C. Meyer, C. Parlitz, and E. Helms, "Powermate : A safe and intuitive robot assistant for handling and assembly tasks," in Proceedings of the 2005 IEEE International Conference on Robotics and Automation, april 2005, pp. 4074-4079.

[15] S. Haddadin, A. Albu-Schäffer, and G. Hirzinger, "Safety evaluation of physical human-robot interaction via crash-testing," in Robotics: Science and Systems Conf. (RSS2007), 2007, pp. 217-224.

[16] M. M. de Graaf and S. Ben Allouch, "Exploring influencing variables for the acceptance of social robots," Robotics and Autonomous Systems, vol. 61, no. 12, pp. 1476-1486, 2013.

[17] M. Mori, “The uncanny valley," Energy, vol. 7, pp. 33-35, 1970.

[18] J. Goetz, S. Kiesler, and A. Powers, "Matching robot appearance and behavior to tasks to improve human-robot cooperation," in Proceedings of the 12th IEEE International Workshop on Robot and Human Interactive Communication, 2003, pp. 55-60.

[19] T. Kanda, T. Miyashita, T. Osada, Y. Haikawa, and H. Ishiguro, "Analysis of humanoid appearances in human-robot interaction," IEEE Transactions on Robotics, vol. 24, no. 3, pp. 725-735, 2008.

[20] D. Kulić and E. Croft, "Affective state estimation for human-robot interaction," IEEE Transactions on Robotics, vol. 23, no. 5, pp. 9911000, oct. 2007.

[21] A. M. Zanchettin, L. Bascetta, and P. Rocco, "Acceptability of robotic manipulators in shared working environments through human-like redundancy resolution," Applied Ergonomics, vol. 44, no. 6, pp. 982-989, 2013.

[22] V. Weistroffer, A. Paljic, L. Callebert, and P. Fuchs, "A methodology to assess the acceptability of human-robot collaboration using virtual reality," in Proceedings of the 19th ACM Symposium on Virtual Reality Software and Technology, 2013, pp. 39-48.

[23] W. Karwowski and M. Rahimi, "Worker selection of safe speed and idle condition in simulated monitoring of two industrial robots," Ergonomics, vol. 34, no. 5, pp. 531-546, 1991.

[24] A. De Santis, G. Di Gironimo, A. Marzano, B. Siciliano, and A. Tarallo, "A virtual-reality-based evaluation environment for wheelchair-mounted manipulators," Eurographics Italian Chapter Conference, vol. 1, pp. 1-8, 2008.

[25] S. Nonaka, K. Inoue, T. Arai, and Y. Mae, "Evaluation of human sense of security for coexisting robots using virtual reality. 1st report: evaluation of pick and place motion of humanoid robots," in Proceedings of the 2004 IEEE International Conference on Robotics and Automation, vol. 3, 2004, pp. 2770-2775.

[26] K. Inoue, S. Nonaka, Y. Ujiie, T. Takubo, and T. Arai, "Comparison of human psychology for real and virtual mobile manipulators," in Proceedings of the 2005 IEEE International Workshop on Robot and Human Interactive Communication, aug. 2005, pp. 73-78.

[27] http://www.technologyreview.com/news/518661/smart-robots-cannow-work-right-next-to-auto workers/.

[28] C. Bartneck, E. Croft, and D. Kulić, "Measurement instruments for the anthropomorphism, animacy, likeability, perceived intelligence, and perceived safety of robots," International Journal of Social Robotics, vol. 1, no. 1, pp. 71-81, 2009. 